\title{
Tumefactive Demyelinating Lesion Differentiated from a Brain Tumor Using a Combination of Magnetic Resonance Imaging and ${ }^{11} \mathrm{C}$-methionine Positron Emission Tomography
}

\author{
Satoko Ninomiya ${ }^{1}$, Makoto Hara ${ }^{1}$, Akihiko Morita ${ }^{1}$, Hiroko Teramoto ${ }^{1}$, Mitsuru Momose ${ }^{2}$, \\ Toshiyuki Takahashi ${ }^{3,4}$ and Satoshi Kamei ${ }^{1}$
}

\begin{abstract}
A 37-year-old woman gradually developed a gait disturbance due to sensory loss in the left lower extremity three years after being diagnosed with clinically isolated syndrome. Brain magnetic resonance imaging (MRI) demonstrated an incomplete ring-enhanced lesion with perifocal edema in the subcortex of the right parietal lobe. ${ }^{11} \mathrm{C}$-methionine positron emission tomography (MET-PET) showed an insignificant uptake in the lesion. The patient was noninvasively diagnosed with tumefactive multiple sclerosis and treated with corticosteroids, and her neurological symptoms and MRI findings improved with treatment. The combination of MRI findings and insignificant uptake on MET-PET is useful for noninvasively differentiating tumefactive demyelinating lesions from brain tumors.
\end{abstract}

Key words: tumefactive multiple sclerosis, cerebral malignant tumors, ${ }^{11} \mathrm{C}$-methionine positron emission tomography, contrast-enhanced MRI

(Intern Med 54: 1411-1414, 2015)

(DOI: 10.2169/internalmedicine.54.3712)

\section{Introduction}

Demyelinating plaques in multiple sclerosis (MS) are generally characterized as multiple, well-demarcated, homogeneous, small ovoid lesions in the white matter of the central nervous system (CNS) $(1,2)$. Tumefactive MS exhibits large (2 $\mathrm{cm}$ or more) demyelinating plaques called tumefactive demyelinating lesions (TDLs), characterized as mass-like lesions with or without ring enhancement on contrastenhanced magnetic resonance imaging (MRI) $(3,4)$. However, the typical radiographic features of large demyelinating plaques often mimic those of brain tumors, particularly malignant tumors, including high-grade gliomas and metastatic brain tumors (5). Moreover, the clinical features of tumefactive MS and brain tumors are similar, and differentiating between these lesions is difficult. Therefore, brain biopsies are often required to confirm the diagnosis (3). ${ }^{11} \mathrm{C}$-methionine positron emission tomography (MET-PET) is widely used as a standard diagnostic modality for detecting brain tumors, especially gliomas $(6,7)$. We herein describe a case of tumefactive MS that was noninvasively diagnosed using a combination of gadolinium-enhanced MRI and MET-PET.

\section{Case Report}

A 37-year-old woman had been followed up for clinically isolated syndrome (CIS) that manifested as left optic neuritis with multiple T2-hyperintense lesions three years previously in periventricular regions on brain MRI. She was admitted to our department due to a gradually progressive gait disturbance and paresthesia in the left lower extremity that had begun two months prior to admission. On admission, the patient's physical examination findings and vital signs were

${ }^{1}$ Division of Neurology, Department of Medicine, Nihon University School of Medicine, Japan, ${ }^{2}$ Department of Diagnostic Imaging and Nuclear Medicine, Tokyo Women's Medical University, Japan, ${ }^{3}$ Department of Neurology, Tohoku University School of Medicine, Japan and ${ }^{4}$ Department of Neurology, Yonezawa National Hospital, Japan

Received for publication July 20, 2014; Accepted for publication October 13, 2014

Correspondence to Dr. Makoto Hara, hara.makoto@nihon-u.ac.jp 
normal. She was alert, and a neurological examination revealed paresthesia and loss of position sense in the left lower extremity. The cranial nerves, muscle power, coordination and deep tendon reflexes in all limbs were normal.

The results of routine blood tests and a urinalysis were within the normal limits. Serology for syphilis, hepatitis B virus, hepatitis $\mathrm{C}$ virus and human immunodeficiency virus was negative, and no anti-aquaporin-4 antibodies were detected on a cell-based assay. The cerebrospinal fluid (CSF) findings were as follows: 1 cell $/ \mathrm{mm}^{3}, 33 \mathrm{mg}$ total protein/ $\mathrm{dL}$, and a slightly increased immunoglobulin $\mathrm{G}$ index of 0.670 (normal $\leq 0.658$ ) (8). Oligoclonal bands were negative, and the level of myelin basic protein was not increased. The cytology of the CSF was class I, and a low-density area with surrounding edema was noted in the subcortex of the right parietal lobe on brain computed tomography. A brain MRI scan also revealed a tumor-like lesion $(2.0 \times 2.6 \mathrm{~cm})$ in the subcortex of the right parietal lobe. The lesion was hypointense on T1-weighted images and hyperintense on fluidattenuated inversion recovery and diffusion-weighted images (Figure A-C). T1-weighted gadolinium-enhanced images also showed an incomplete ring-enhanced lesion (Figure D-F), and an insignificant methionine uptake was observed on MET-PET, including in the tumor-like lesion detected on MRI (Figure G-I). The tumor to normal contralateral frontal MET uptake ratio (T/N ratio) of the tumor-like lesion was 1.51 , and a whole-body investigation for malignant tumors was negative. We clinically diagnosed the patient with MS according to the 2010 McDonald criteria for MS (9) based on her past history of CIS and newly detected enhanced lesions on brain MRI. The tumor-like lesion was noninvasively diagnosed as TDL using a combination of contrast-enhanced MRI and MET-PET.

The patient was treated with intravenous methylprednisolone $(1,000 \mathrm{mg} / \mathrm{day})$ for three days in addition to high-dose oral prednisolone (1 mg/kg/day). The dose of prednisolone was tapered by $10 \mathrm{mg}$ every five days and then discontinued. Consequently, the paresthesia in the left lower extremity and gait disturbance remarkably improved, and she was discharged after 14 days on foot. Brain MRI performed at the 12-month follow-up demonstrated that the TDL in the right parietal lobe had markedly decreased in size and no longer exhibited gadolinium enhancement (Figure J-L). The patient has been free of neurological symptoms for 18 months.

\section{Discussion}

Tumefactive MS patients present with various symptoms, depending on the location and size of the lesion (3). The clinical findings of TDL include a rapidly progressive clinical course. However, it is not rare for the duration from the onset of symptoms to admission to be more than one month (10). Indeed, the TDL in the present case gradually progressed to a gait disturbance and paresthesia of the left lower extremity over two months. The variable clinical find- ings and disease course may result in an incorrect or delayed diagnosis and treatment. Therefore, it is crucial to confirm the correct diagnosis as an inflammatory demyelinating disease.

The characteristics of TDL on brain MRI include solitary large lesions measuring $>2 \mathrm{~cm}$ in size associated with the mass effect, perifocal edema and/or the presence of ring enhancement $(3,4)$ that is often incomplete or open, called open-ring enhancement (11). The detection of incomplete or open-ring enhanced lesions on MRI is particularly considered to be a specific finding for TDL (3), and these characteristic findings were noted in the present case. Pallud et al. (12) reviewed 927 histologically proven WHO grade II (low-grade) gliomas for which contrast-enhanced MR images were available and found that none of the lesions showed a ring-enhanced pattern, although the WHO grade III and IV (high-grade) gliomas demonstrated ring enhancement on MRI. Kim et al. (13) also reviewed 15 patients with TDL and 48 patients with primary brain tumors and reported that MRI alone does not provide sufficiently high diagnostic accuracy for distinguishing TDLs from gliomas or lymphomas. Hence, brain biopsies are frequently required to confirm the diagnosis of TDL and differentiate these lesions from brain tumors $(3,5)$.

MET-PET is widely used as a standard diagnostic modality for assessing brain tumors, particularly high-grade gliomas $(6,7)$, as MET-PET has high sensitivity and specificity for detecting high-grade brain tumors, especially gliomas (6). It has been reported that it is possible to differentiate high-grade gliomas from non-neoplastic lesions, with a reported sensitivity of $93 \%$ and specificity of $78 \%$ when the $\mathrm{T} / \mathrm{N}$ ratio is over $2.0(14)$. The $\mathrm{T} / \mathrm{N}$ ratio in the present case was 1.51; therefore, the high-grade glioma could be differentiated. However, low-grade gliomas sometimes cannot be differentiated from non-neoplastic lesions using only MET-PET, as the $\mathrm{T} / \mathrm{N}$ ratios of these types of lesions overlap (14). When the cut-off for the $\mathrm{T} / \mathrm{N}$ ratio is set to 1.47 , a sensitivity of $56 \%$ and specificity of $30 \%$ have been reported for differentiating low-grade gliomas from nonneoplastic lesions (14). In addition, methionine uptake is detected in certain stages of non-tumor CNS diseases, including brain abscesses (15) and the acute inflammatory phase of MS $(14,15)$.

Kawai et al. (14) described four cases of MS without TDL (typical MS) examined with MET-PET in which the demyelinating lesions showed no uptake compared with normal brain regions, with the exception of one case of a mild methionine uptake (T/N ratio: 1.49). Padma et al. also reported a case of typical MS with a mild methionine uptake on MET-PET (15). These reports suggest that the detection of an insignificant uptake in typical MS lesions on METPET may be useful for distinguishing these lesions from brain tumors. The TDL in the present case showed an insignificant uptake on MET-PET, indicating that the lesion was unlikely a high-grade malignancy. The patient's past history of optic neuritis and favorable response to corticosteroids 

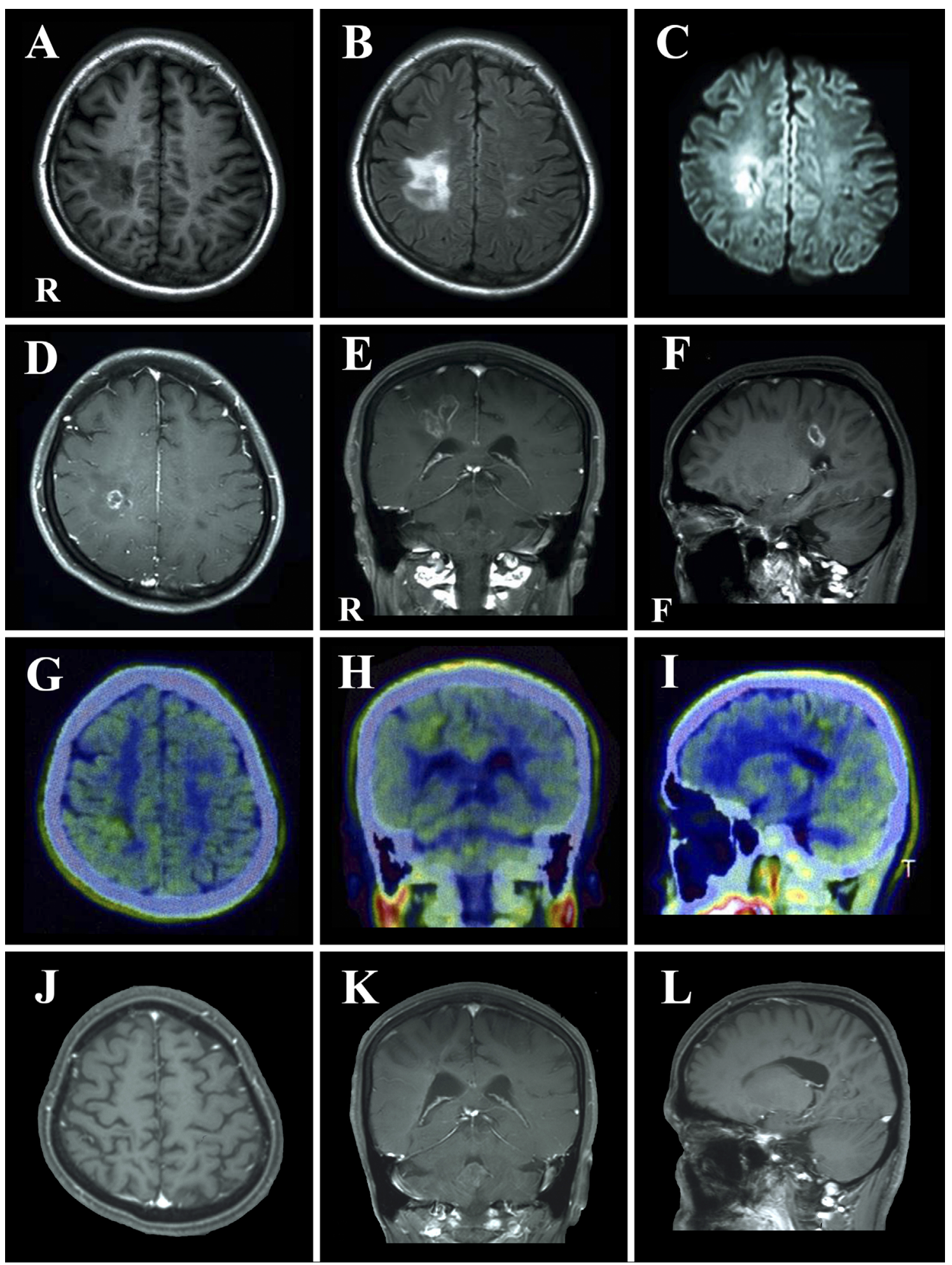

Figure. Tumefactive demyelinating lesion on brain MRI and MET-PET. On brain MRI, axial T1weighted images (A) demonstrated a hypointense lesion in the subcortex of the right parietal lobe. The lesion was hyperintense on a fluid-attenuated inversion recovery image $(B)$ and diffusion-weighted image (C). T1-weighted images with gadolinium enhancement on axial (D), coronal (E) and sagittal (F) sections demonstrated incomplete open-ring enhancement with a mass effect. On MET-PET, an insignificant methionine uptake was noted, including in the gadolinium-enhanced lesion (T/N ratio: 1.51) (G-I). At the 1-year follow-up, the lesion was not enhanced on T1-weighted MRI with gadolinium enhancement $(\mathrm{J}-\mathrm{L})$.

are also consistent with the diagnosis of TDL.

Recently, a combination of radiographic and functional/ metabolic imaging modalities has been used to diagnose TDL (15) in order to avoid unnecessary surgery. Selkirk et 
al. (16) described the features of TDL, including the presence of mass-like ring-enhanced lesions on MRI and hypometabolic masses on ${ }^{18} \mathrm{~F}$-fluorodeoxyglucose-PET, features that are not suggestive of high-grade malignancy. Finally, the authors noninvasively diagnosed a ring-enhanced mass on MRI as a TDL coupled with responsiveness to corticosteroids. Similarly, multimodality testing with contrastenhanced MRI and MET-PET, as performed in the current case, showing a hypometabolic MET-PET lesion with incomplete ring enhancement on MRI, is useful for differentiating tumefactive MS from brain tumors.

To the best of our knowledge, this is the first case report to efficiently differentiate tumefactive MS from a brain tumor using a combination of contrast-enhanced MRI and MET-PET. The use of this combination of imaging modalities may preclude the need for brain biopsies in select cases.

The authors state that they have no Conflict of Interest (COI).

\section{Financial Support}

This work was supported by a grant from the MEXTSupported Program for the Strategic Research Foundation at Private Universities, 2014 (S1291004).

\section{References}

1. Noseworthy JH, Lucchinetti C, Rodriguez M, Weinshenker BG Multiple sclerosis. N Engl J Med 343: 938-952, 2000.

2. Horowitz AL, Kaplan RD, Grewe G, White RT, Salberg LM. The ovoid lesion: a new MR observation in patients with multiple sclerosis. Am J Neuroradiol 10: 303-305, 1989.

3. Lucchinetti CF, Gavrilova RH, Metz I, et al. Clinical and radiographic spectrum of pathologically confirmed tumefactive multiple sclerosis. Brain 131: 1759-1775, 2008.

4. Dagher AP, Smirniotopoulos J. Tumefactive demyelinating lesions.
Neuroradiology 38: 560-565, 1996.

5. Di Patre PL, Castillo V, Delavelle J, Vuillemoz S, Picard F, Landis T. "Tumor-mimicking" multiple sclerosis. Clin Neuropathol 22: 235-239, 2003

6. Singhal T, Narayanan TK, Jain V, Mukherjee J, Mantil J. 11C-Lmethionine positron emission tomography in the clinical management of cerebral gliomas. Mol Imaging Biol 10: 1-18, 2008.

7. Kato T, Shinoda J, Nakayama N, et al. Metabolic assessment of gliomas using $11 \mathrm{C}$-methionine, $[18 \mathrm{~F}]$ fluorodeoxyglucose, and 11 C-choline positron-emission tomography. Am J Neuroradiol 29: 1176-1182, 2008.

8. Kira J, Kanai T, Nishimura Y, et al. Western versus Asian types of multiple sclerosis: immunogenetically and clinically distinct disorders. Ann Neurol 40: 569-574, 1996.

9. Polman CH, Reingold SC, Banwell B, et al. Diagnostic criteria for multiple sclerosis: 2010 revisions to the McDonald criteria. Ann Neurol 69: 292-302, 2011.

10. Xia L, Lin S, Wang ZC, et al. Tumefactive demyelinating lesions: nine cases and a review of the literature. Neurosurg Rev 32: 171179, 2009.

11. Masdeu JC, Quinto C, Olivera C, Tenner M, Leslie D, Visintainer P. Open-ring imaging sign: highly specific for atypical brain demyelination. Neurology 54: 1427-1433, 2000.

12. Pallud J, Capelle L, Taillandier L, et al. Prognostic significance of imaging contrast enhancement for WHO grade II gliomas. Neuro Oncol 11: 176-182, 2009.

13. Kim DS, Na DG, Kim KH, et al. Distinguishing tumefactive demyelinating lesions from glioma or central nervous system lymphoma: added value of unenhanced CT compared with conventional contrast-enhanced MR imaging. Radiology 251: 467-475, 2009.

14. Kawai N, Okauchi M, Miyake K, et al. 11C-methionine positron emission tomography in nontumorous brain lesions. No Shinkei Geka (Neurological Surgery) 38: 985-995, 2010 (in Japanese, Abstract in English).

15. Padma MV, Adineh M, Pugar K, et al. Functional imaging of a large demyelinating lesion. J Clin Neurosci 12: 176-178, 2005.

16. Selkirk SM, Shi J. Relapsing-remitting tumefactive multiple sclerosis. Mult Scler 11: 731-734, 2005.

(C) 2015 The Japanese Society of Internal Medicine http://www.naika.or.jp/imonline/index.html 